\title{
Dynamics and Control for Nonholonomic Mobile Modular Manipulators
}

\author{
Yangmin Li \& Yugang Liu \\ University of Macau \\ Macao S. A.R., P. R. China
}

\section{Introduction}

The development of a robot requires that it be able to adopt as many configurations as possible using limited modules, so as to allow the construction of new types of robots without redesign and remanufacturing. Traditionally, modular manipulators are mounted on a fixed base whose mobility is constrained. However, with the development of industry and technology, such modular manipulators as mounted on fixed bases can not meet some practical requirements any more. An intelligent and autonomous mobile manipulator, which can fulfil some operations without human interference, has become an active research topic recently since it has many potential applications such as in modern factories for transporting materials, in dangerous fields for dismantling bombs or moving nuclear infected objects, in modern families for doing housework, as well as in the public places for city maintenance.

In this chapter, a nonholonomic mobile platform is attached to the modular manipulator in order to increase workspace of the entire robot. Building up the dynamic model for a nonholonomic mobile modular manipulator is a challenging task due to the interactive motions between the modular manipulator and the mobile platform, as well as the nonholonomic constraints of the mobile platform. Also a trajectory following task becomes even more complex and difficult to achieve.

Such conventional control strategies as computed-torque control require precise apriori knowledge of the dynamic parameters for the controlled system. However, in practical applications, it is almost impossible to obtain exact dynamic parameters for a mobile modular manipulator because of such uncertainties as complex nonlinear frictions, flexibilities of the joints and links, payload variations, and terrain irregularities. Robust control techniques provide a natural rejection to external disturbances, which are provided by a high-frequency commuted control action that constrains the error $\pi$ trajectories to stay on the sliding surface. Classical sliding mode control law adopts sign of functions and the caused chattering may do harm to the robots. Adaptive control \& technique does not rely on precise apriori knowledge of dynamic parameters and it can suppress such errors as caused by parameter uncertainties by online adjusting dynamic parameters. Furthermore, adaptive control can counteract the negative influence of highfrequency switching caused by robust control because its action has naturally smooth time behaviour. 
In related work on modular robots, the modular robot concept could be traced back to the 1970's (Will \& Grossman, 1975). In early modular robot research, the emphasis was put on the structure design of self-organizing, self-reconfigurable, self-assembling, and selfrepairable modular robots (Fukuda et al., 1989; Tomita et al., 1999). Kinematic and dynamic analysis as well as trajectory planning became another active topic in the past decades (Chen \& Yang, 1998; Fei et al., 2001). In recent years, the scholars had turned their attentions to trajectory following control for modular manipulators (Melek \& Goldenberg, 2003; Shen et al., 2002; Stoy et al., 2002). Parameter identification and vibration control for a 9-DOF reconfigurable modular manipulator were investigated by authors in ( $\mathrm{Li}$ et al., 2004a).

Regarding to literatures on mobile manipulators, mobile manipulators were exploited to install and remove aircraft warning spheres (Campos et al., 2002), to polish aircraft canopy (Jamisola et al., 2002), to organize furniture in a room (Rus et al., 1995), and to collectively transport a single palletized load (Stilwell \& Bay, 1993). A great deal of research activities can be found on motion planning of mobile manipulators (Carriker et al., 1991; Chitta \& Ostrowski, 2002; Nagatani et al., 2002). Several kinematic and dynamic modelling methods were presented for mobile manipulators in the past decade, such as the Kane's method (Tanner \& Kyriakopoulos, 2001), the Newton-Euler method (Chung \& Velinsky, 1999) and the Lagrange method (Li \& Liu, 2004b; Liu \& Li, 2005a; Yu \& Chen, 2002). Tip-over analysis and prevention attracted numerous scholars and several tip-over stability criteria were defined, such as the potential energy stability level (Ghasempoor \& Sepehri, 1995), the force-angle stability measure (Papadopoulos \& Rey, 1996), the zero moment point criterion (Furuno et al., 2003), and the criterion based on supporting forces (Li \& Liu, 2005b). Extensive literatures can be found on control of mobile manipulators. Dynamic characteristics between the mobile platform and the onboard manipulator were investigated (Yamamoto \& Yun, 1996). A robust control method was developed to eliminate the harmful effect of the wheel slip on the tracking performance of a spatial mobile manipulator (Chung \& Velinsky, 1999). A homogeneous kinematic stabilization strategy and an adaptive control scheme were combined for mobile manipulator control without any knowledge of the system dynamic model (Colbaugh, 1998). Neural network and fuzzy logic control for mobile manipulators were also studied by authors (Li \& Liu, 2005c, 2005d, 2006a, 2006b).

In the previous research work, modeling for the mobile platform and for the manipulator was usually carried out separately and control for nonholonomic mobile robots was mostly limited to kinematic velocity control, while few work on dynamic torque control. The interactive motions between the manipulator and the mobile platform made the models established inaccurate, which then affected the control result. Most of the present controllers were designed in joint space, but few in task space (Ge et al., 1997). However, in practical applications, the end-effector of a robot is usually specified to fulfil some operations.

This chapter is organized as follows: an integrated modelling method is proposed considering nonholonomic constraints and interactive motions in Section 2. In Section 3, a robust adaptive controller is designed in task space to control the end-effector to follow desired spatial trajectories. Simulations are conducted on a real mobile modular manipulator, and a comparison is made with the conventional model-based controller in Section 4. Section 5 gives some concluding remarks. Some suggested ideas for future research work are presented in the last section. 


\section{An Integrated Modelling Method}

\subsection{Kinematics analysis}

A mobile modular manipulator is normally composed of an $m$-wheeled holonomic or nonholonomic mobile platform and an $n$-DOF onboard modular manipulator, as shown in Fig. 1(a). In this chapter, we analyze the 3-wheeled nonholonomic mobile platform, which has two driving wheels and one castor wheel. The two driving wheels are coaxial and mounted in front of the platform and the castor wheel in the rear is orientable with respect to the cart. The robot is assumed to move on a horizontal plane; then the motion of the mobile platform can be illustrated as shown in Fig. 1(b). An arbitrary inertial base frame $O_{B} X_{B} Y_{B} Z_{B}$ is fixed on the motion plane, while a frame $O_{m} X_{m} Y_{m} Z_{m}$ is attached to the mobile platform. In frame $O_{m} X_{m} Y_{m} Z_{m}, O_{m}\left(x_{m}, y_{m}\right)$ is selected as the midpoint of the line segment connecting the two driving-wheel centres; $O_{m} Y_{m}$ is along the coaxial of the two driving wheels; $O_{m} X_{m}$ is perpendicular to $O_{m} Y_{m}$ and passes through $O_{m}$. The heading angle $\phi_{m}$ determines the posture of the mobile platform.

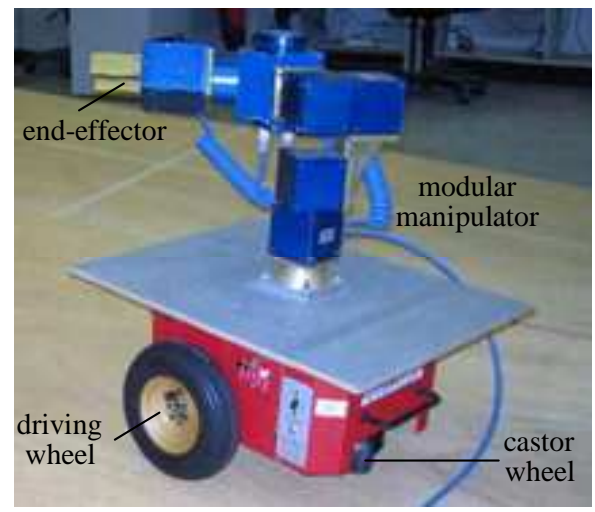

(a) Prototype

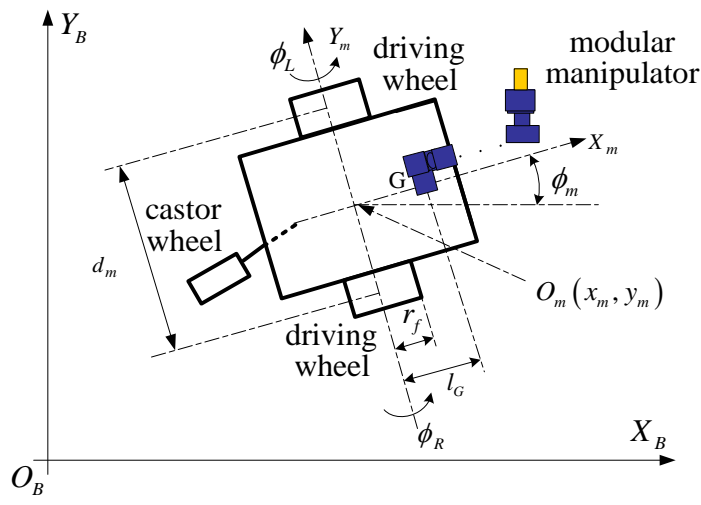

(b) coordinate system definition

Fig. 1. Prototype and coordinate system definition for a mobile modular manipulator. 
Since the modular manipulator is mounted on the mobile platform, it can be assumed that the relative position of frame $O_{m} X_{m} Y_{m} Z_{m}$ and the frame $O_{0} X_{0} Y_{0} Z_{0}$ is steady; here $O_{0} X_{0} Y_{0} Z_{0}$ is the frame attached to the $1^{\text {st }}$ module of the modular manipulator. Therefore the mobile platform can be treated as a special module added to the bottom of the modular manipulator, which can both move on the motion plane and rotate about the vertical axis. From Fig. 1(b), transformation matrices between the frames $O_{B} X_{B} Y_{B} Z_{B}, O_{m} X_{m} Y_{m} Z_{m}$ and $O_{0} X_{0} Y_{0} Z_{0}$ are given by

$$
{ }_{m}^{B} T=\left[\begin{array}{cccc}
\cos \phi_{m} & -\sin \phi_{m} & 0 & x_{m} \\
\sin \phi_{m} & \cos \phi_{m} & 0 & y_{m} \\
0 & 0 & 1 & r_{f} \\
0 & 0 & 0 & 1
\end{array}\right], \quad{ }_{0}^{m} T=\left[\begin{array}{cccc}
1 & 0 & 0 & l_{G} \\
0 & 1 & 0 & 0 \\
0 & 0 & 1 & h_{G} \\
0 & 0 & 0 & 1
\end{array}\right]
$$

where $r_{f}$ is the radius for the driving wheels; $l_{G}$ and $h_{G}$ denote offsets of the $1^{\text {st }}$ module with respect to $O_{m} X_{m} Y_{m} Z_{m}$ along $O_{m} X_{m}, O_{m} Y_{m}$ respectively.

By defining the link four parameters according to the Denavit-Hartenberg (D.H.) notation (de Wit et al., 1996), the transformation matrix between two adjacent links for the modular manipulator can be derived as follows

$$
{ }_{i}^{i-1} T=\left[\begin{array}{cccc}
\cos q_{i} & -\sin q_{i} & 0 & l_{i} \\
\sin q_{i} \cos \alpha_{i} & \cos q_{i} \cos \alpha_{i} & -\sin \alpha_{i} & -d_{i} \sin \alpha_{i} \\
\sin q_{i} \sin \alpha_{i} & \cos q_{i} \sin \alpha_{i} & \cos \alpha_{i} & d_{i} \cos \alpha_{i} \\
0 & 0 & 0 & 1
\end{array}\right]
$$

where $l_{i}, \alpha_{i}, d_{i}, q_{i}$ are D.H. parameters.

Therefore the transformation matrix of the end effector with respect to the inertial base frame can be derived by

$$
{ }_{n}^{B} T={ }_{0}^{B} T_{1}^{0} T{ }_{2}^{1} T \cdots{ }_{n}^{n-1} T
$$

Hence, the position vector $\vec{p}_{e}$ and the posture vectors $\vec{n}, \vec{o}, \vec{a}$ of the end-effector with respect to the inertial base frame can be derived, which can be observed from the fourth column and the first three columns of the matrix ${ }_{n}^{B} T$ correspondingly.

However, it is inconvenient to describe the posture of the end-effector using these posture vectors with nine parameters. The posture can be determined by only three independent parameters using Z- $Y-Z$ Euler angles $\phi, \theta$ and $\psi$. Relationship between the posture vectors and the Euler angles can be described as follows

$$
\begin{aligned}
& \phi=a \tan 2\left(a_{y}, a_{x}\right), \\
& \theta=a \tan 2\left(o_{z},-n_{z}\right), \\
& \psi=a \tan 2\left(a_{x} \cos \phi+a_{y} \sin \phi, a_{z}\right)
\end{aligned}
$$




\subsection{Nonholonomic constraints and Jacobian}

The motion of a mobile modular manipulator during a very short time interval $\left[\begin{array}{ll}t^{i} & t^{i+1}\end{array}\right]$ is shown in Fig. 2. $l_{r}, d_{r}$ and $d_{m}$ represent the distances from the fixed bar to $O_{m} Y_{m}$, from the fixed bar to the horizontal axis of the castor wheel, and that between the two driving wheels respectively; $r_{i}$ and $\theta_{i}$ are steering radius and yaw angle during the time interval; $\phi_{L^{\prime}} \phi_{R^{\prime}} \phi_{r}$ and $\beta_{r}$ denote rotating angles of the left driving wheel, the right driving wheel, the castor wheel around its horizontal axis, and that around the fixed bar respectively; $\Delta S_{L}, \Delta S_{R}$ and $\Delta S_{m}$ represent advances of the left driving wheel, the right driving wheel, and the mobile platform during the time interval correspondingly.

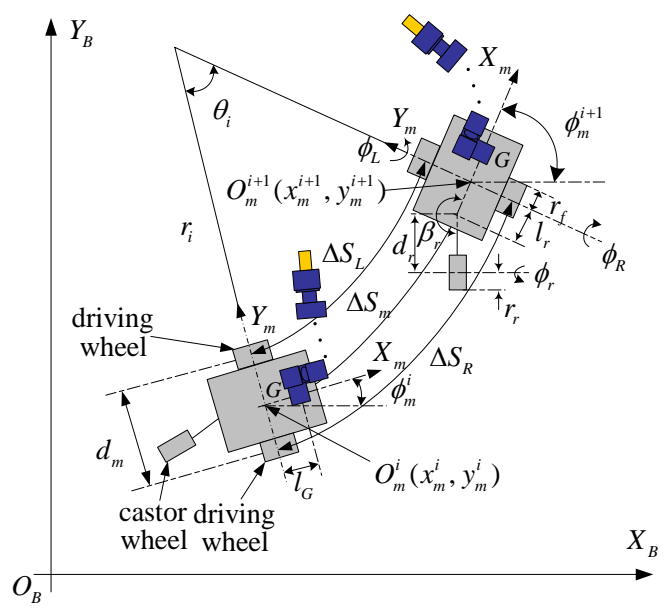

Fig. 2. Nonholonomic constraints derivation.

Let the time interval $\Delta t=t^{i+1}-t^{i} \rightarrow 0$, on the assumption of low speeds, velocities during this time interval can be treated as constants. Then,

$$
\left\{\begin{aligned}
\Delta x_{m}^{i} & =\left|O_{m}^{i} O_{m}^{i+1}\right| \cdot \cos \phi_{m}^{i} \\
\Delta y_{m}^{i} & =\left|O_{m}^{i} O_{m}^{i+1}\right| \cdot \sin \phi_{m}^{i} \\
\Delta S_{L} & =\theta_{i} \cdot\left(r_{i}-\frac{d_{m}}{2}\right) \\
\Delta S_{R} & =\theta_{i} \cdot\left(r_{i}+\frac{d_{m}}{2}\right) \\
\Delta S_{m} & \approx\left|O_{m}^{i} O_{m}^{i+1}\right| \\
\theta_{i} & \approx \Delta \phi_{m}^{i} \\
r_{i} & =\frac{\Delta S_{m}}{\theta_{i}}
\end{aligned}\right.
$$


where $\Delta x_{m}^{i}=x_{m}^{i+1}-x_{m}^{i}, \Delta y_{m}^{i}=y_{m}^{i+1}-y_{m}^{i}$ and $\Delta \phi_{m}^{i}=\phi_{m}^{i+1}-\phi_{m}^{i}$.

Substituting Eq. 5(f, g) into Eq. 5(c, d) yields

$$
\left\{\begin{array}{l}
\Delta S_{L}=\Delta S_{m}-\frac{d_{m}}{2} \cdot \Delta \phi_{m}^{i} \\
\Delta S_{R}=\Delta S_{m}+\frac{d_{m}}{2} \cdot \Delta \phi_{m}^{i}
\end{array}\right.
$$

Adding Eq. 6(a) and Eq. 6(b) yields

$$
\Delta S_{m}=\frac{\Delta S_{L}+\Delta S_{R}}{2}
$$

Subtracting Eq. 6(a) for Eq. 6(b) yields

$$
\Delta \phi_{m}=-\frac{\Delta S_{L}-\Delta S_{R}}{d_{m}}
$$

Substituting Eq. 7 and Eq. 5(e) into Eq. 5(a,b) yields

$$
\left\{\begin{array}{l}
\Delta x_{m}^{i}=\frac{\cos \phi_{m}^{i}}{2} \cdot\left(\Delta S_{L}+\Delta S_{R}\right) \\
\Delta y_{m}^{i}=\frac{\sin \phi_{m}^{i}}{2} \cdot\left(\Delta S_{L}+\Delta S_{R}\right)
\end{array}\right.
$$

From Eq. 8 and Eq. 9,

$$
\left\{\begin{array}{l}
\dot{x}_{m}^{i}=\lim _{\Delta t \rightarrow 0}\left(\frac{\Delta x_{m}^{i}}{\Delta t}\right)=\frac{\cos \phi_{m}^{i}}{2} \cdot\left[\lim _{\Delta t \rightarrow 0}\left(\frac{\Delta S_{L}}{\Delta t}\right)+\lim _{\Delta t \rightarrow 0}\left(\frac{\Delta S_{R}}{\Delta t}\right)\right]=\frac{\cos \phi_{m}^{i}}{2} \cdot\left(\dot{S}_{L}+\dot{S}_{R}\right) \\
\dot{y}_{m}^{i}=\lim _{\Delta t \rightarrow 0}\left(\frac{\Delta y_{m}^{i}}{\Delta t}\right)=\frac{\sin \phi_{m}^{i}}{2} \cdot\left[\lim _{\Delta t \rightarrow 0}\left(\frac{\Delta S_{L}}{\Delta t}\right)+\lim _{\Delta t \rightarrow 0}\left(\frac{\Delta S_{R}}{\Delta t}\right)\right]=\frac{\sin \phi_{m}^{i}}{2} \cdot\left(\dot{S}_{L}+\dot{S}_{R}\right) \\
\dot{\phi}_{m}^{i}=\lim _{\Delta t \rightarrow 0}\left(\frac{\Delta \phi_{m}^{i}}{\Delta t}\right)=-\frac{1}{d_{m}} \cdot\left[\lim _{\Delta t \rightarrow 0}\left(\frac{\Delta S_{L}}{\Delta t}\right)-\lim _{\Delta t \rightarrow 0}\left(\frac{\Delta S_{R}}{\Delta t}\right)\right]=-\frac{1}{d_{m}} \cdot\left(\dot{S}_{L}-\dot{S}_{R}\right)
\end{array}\right.
$$

Equation 10 always holds during the course of plane motion, so the superscript " $\mathrm{i}$ " can be omitted. Considering that $\dot{S}_{L}=r_{f} \cdot \dot{\phi}_{L}$ and $\dot{S}_{R}=r_{f} \cdot \dot{\phi}_{R}$, we can obtain

$$
\left\{\begin{array}{l}
\dot{x}_{m}=\frac{r_{f} \cdot \cos \phi_{m}}{2} \cdot \dot{\phi}_{L}+\frac{r_{f} \cdot \cos \phi_{m}}{2} \cdot \dot{\phi}_{R} \\
\dot{y}_{m}=\frac{r_{f} \cdot \sin \phi_{m}}{2} \cdot \dot{\phi}_{L}+\frac{r_{f} \cdot \sin \phi_{m}}{2} \cdot \dot{\phi}_{R} \\
\dot{\phi}_{m}=-\frac{r_{f}}{d_{m}} \cdot \dot{\phi}_{L}+\frac{r_{f}}{d_{m}} \cdot \dot{\phi}_{R}
\end{array}\right.
$$


In the same way, we can obtain

$$
\left\{\begin{array}{l}
\dot{\phi}_{r}=\frac{\cos \left(\beta_{r}+\phi_{m}\right)}{r_{r}} \cdot \dot{x}_{m}+\frac{\sin \left(\beta_{r}+\phi_{m}\right)}{r_{r}} \cdot \dot{y}_{m}-\frac{l_{r} \sin \beta_{r}}{r_{r}} \cdot \dot{\phi}_{m} \\
\dot{\beta}_{r}=\frac{\sin \left(\beta_{r}+\phi_{m}\right)}{d_{r}} \cdot \dot{x}_{m}-\frac{\cos \left(\beta_{r}+\phi_{m}\right)}{d_{r}} \cdot \dot{y}_{m}-\frac{d_{r}-l_{r} \cos \beta_{r}}{d_{r}} \cdot \dot{\phi}_{m}
\end{array}\right.
$$

Adding Eq. 11(a) multiplied by $\cos \phi_{m}$ to Eq. 11(b) multiplied by $\sin \phi_{m}$ yields

$$
\cos \phi_{m} \cdot \dot{x}_{m}+\sin \phi_{m} \cdot \dot{y}_{m}=\frac{r_{f}}{2} \cdot \dot{\phi}_{L}+\frac{r_{f}}{2} \cdot \dot{\phi}_{R}
$$

Adding Eq. 11(c) multiplied by $\frac{d_{m}}{2}$ to Eq. 13 yields

$$
\cos \phi_{m} \cdot \dot{x}_{m}+\sin \phi_{m} \cdot \dot{y}_{m}+\frac{d}{2} \cdot \dot{\phi}_{m}-r_{f} \cdot \dot{\phi}_{R}=0
$$

Subtracting Eq. 11(c) multiplied by $\frac{d_{m}}{2}$ from Eq. 13 yields

$$
\cos \phi_{m} \cdot \dot{x}_{m}+\sin \phi_{m} \cdot \dot{y}_{m}-\frac{d_{m}}{2} \cdot \dot{\phi}_{m}-r_{f} \cdot \dot{\phi}_{L}=0
$$

Subtracting Eq. 11(b) multiplied by $\cos \phi_{m}$ from Eq. 11(a) multiplied by $\sin \phi_{m}$ yields

$$
\sin \phi_{m} \cdot \dot{x}_{m}-\cos \phi_{m} \cdot \dot{y}_{m}=0
$$

From Eq. 12 and Eq. 14-16, we have

$$
\left[\begin{array}{ccccccc}
\cos \phi_{m} & \sin \phi_{m} & -\frac{d_{m}}{2} & 0 & 0 & -r_{f} & 0 \\
\cos \phi_{m} & \sin \phi_{m} & \frac{d_{m}}{2} & 0 & 0 & 0 & -r_{f} \\
\sin \phi_{m} & -\cos \phi_{m} & 0 & 0 & 0 & 0 & 0 \\
\cos \left(\beta_{r}+\phi_{m}\right) & \sin \left(\beta_{r}+\phi_{m}\right) & -l_{r} \sin \beta_{r} & -r_{r} & 0 & 0 & 0 \\
\sin \left(\beta_{r}+\phi_{m}\right) & -\cos \left(\beta_{r}+\phi_{m}\right) & l_{r} \cos \beta_{r}-d_{r} & 0 & -d_{r} & 0 & 0
\end{array}\right] \cdot\left[\begin{array}{c}
\dot{x}_{m} \\
\dot{y}_{m} \\
\dot{\phi}_{m} \\
\dot{\phi}_{r} \\
\dot{\beta}_{r} \\
\dot{\phi}_{L} \\
\dot{\phi}_{R}
\end{array}\right]=0
$$

in short $A(\xi) \cdot \dot{\xi}=0$; here $\xi=\left[\begin{array}{lllllll}x_{m} & y_{m} & \phi_{m} & \phi_{r} & \beta_{r} & \phi_{L} & \phi_{R}\end{array}\right]^{T}$.

From Eq. 11 and Eq. 12, we can easily obtain 


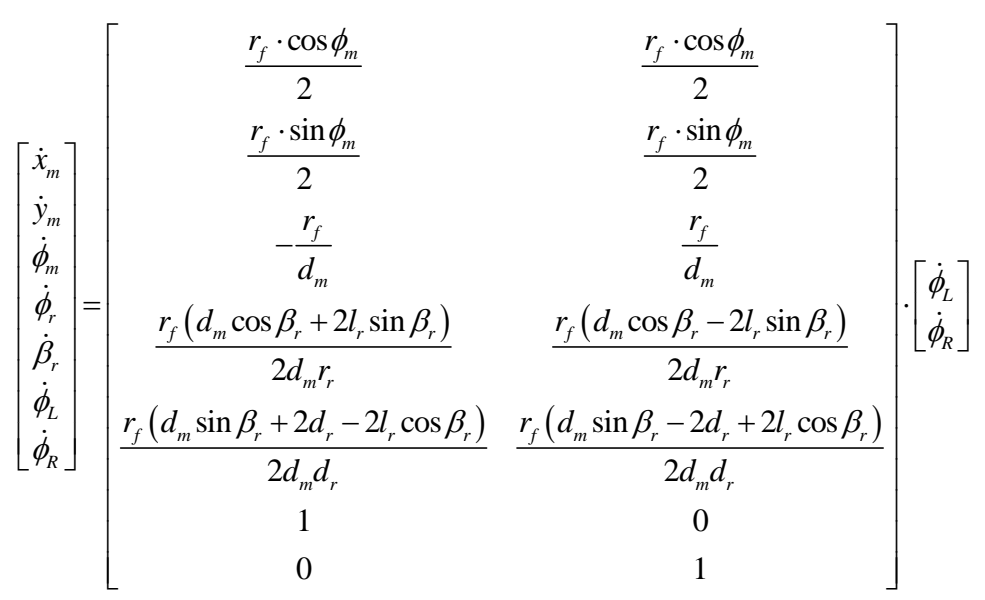

in short $\dot{\xi}=S(\xi) \cdot\left[\begin{array}{ll}\dot{\phi}_{L} & \dot{\phi}_{R}\end{array}\right]^{T}$.

Substituting Eq. 18 into Eq. 17 yields

$$
A(\xi) \cdot S(\xi)=0
$$

Let $x=\left[\begin{array}{llllll}p_{x} & p_{y} & p_{z} & \phi & \theta & \psi\end{array}\right]^{T}$ and $q=\left[\begin{array}{llllll}\phi_{L} & \phi_{R} & q_{1} & q_{2} & \cdots & q_{n}\end{array}\right]^{T}$ be coordinates in task space and joint space respectively; and define $\varsigma_{\zeta}=\left[\begin{array}{llll}\xi^{T} & q_{1} & \cdots & q_{n}\end{array}\right]^{T}$ as extended coordinates, and then the Jacobian matrix can be derived by

$$
J=\frac{\partial x}{\partial q}=\frac{\partial x}{\partial \varsigma} \cdot\left[\begin{array}{cc}
S(\xi) & 0_{7 \times n} \\
0_{n \times 2} & I_{n \times n}
\end{array}\right]
$$

\subsection{Dynamic modelling}

With the assumption of moving on a horizontal plane, the mobile platform has a constant potential energy $U_{m}$. To calculate the kinetic energy, the mobile platform can be divided into four parts, the cart (including all the driving units in the box), the left front wheel, the right front wheel and the rear wheel, that is

$$
T_{m}=T_{c}+T_{l f}+T_{r f}+T_{r}
$$

where $T_{m}$ is the entire kinetic energy for the mobile platform; $T_{c}, T_{l f}, T_{r f}$ and $T_{r}$ denote respectively kinematic energy of the cart, the left and right front wheel and the rear wheel. Kinetic energy for the cart can be calculated by

$$
T_{c}=\frac{1}{2} \cdot m_{c} \cdot \dot{x}_{c}^{2}+\frac{1}{2} \cdot m_{c} \cdot \dot{y}_{c}^{2}+\frac{1}{2} \cdot I_{c} \cdot \dot{\phi}_{m}^{2}
$$

where $m_{c}$ is the mass of the cart; $x_{c}=x_{m}+l_{c} \cos \phi_{m}, y_{c}=y_{m}+l_{c} \sin \phi_{m}$ denote coordinates for the mass centre of the cart, with $l_{c}$ being distance between the mass centre and $O_{m} ; I_{c}$ represents the moment of inertia for the cart around the axis of $O_{m} Z_{m}$. 
With the assumption of rolling without slipping, the contact points of the wheels with the motion plane can be treated as instant rotating centres. Then, kinematic energy for the front driving wheels can be given by

$$
T_{l f}=\frac{3}{4} \cdot m_{f} \cdot r_{f}^{2} \cdot \dot{\phi}_{L}^{2}, \quad T_{r f}=\frac{3}{4} \cdot m_{f} \cdot r_{f}^{2} \cdot \dot{\phi}_{R}^{2}
$$

where $m_{f}$ is the mass for the front driving wheels.

The castor wheel can rotate about both its axis and the fixed bar that does not pass through its centre, as shown in Fig. 3. So, the kinetic energy of the rear castor wheel includes two parts as given by Eq. 24 .

$$
T_{r}=\frac{1}{2} \cdot I_{r 1} \cdot \dot{\phi}_{r}^{2}+\frac{1}{2} \cdot I_{r 2} \cdot \dot{\beta}_{r}^{2}
$$

where the moments of inertia $I_{r 1}$ and $I_{r 2}$ can be determined by

$$
\begin{aligned}
& I_{r 1}=\frac{1}{2} \cdot m_{r} \cdot r_{r}^{2}+m_{r} \cdot r_{r}^{2}=\frac{3}{2} \cdot m_{r} \cdot r_{r}^{2} \\
& I_{r 2}=\frac{1}{4} \cdot m_{r} \cdot r_{r}^{2}+m_{r} \cdot d_{r}^{2}=m_{r} \cdot\left(\frac{r_{r}^{2}}{4}+d_{r}^{2}\right)
\end{aligned}
$$

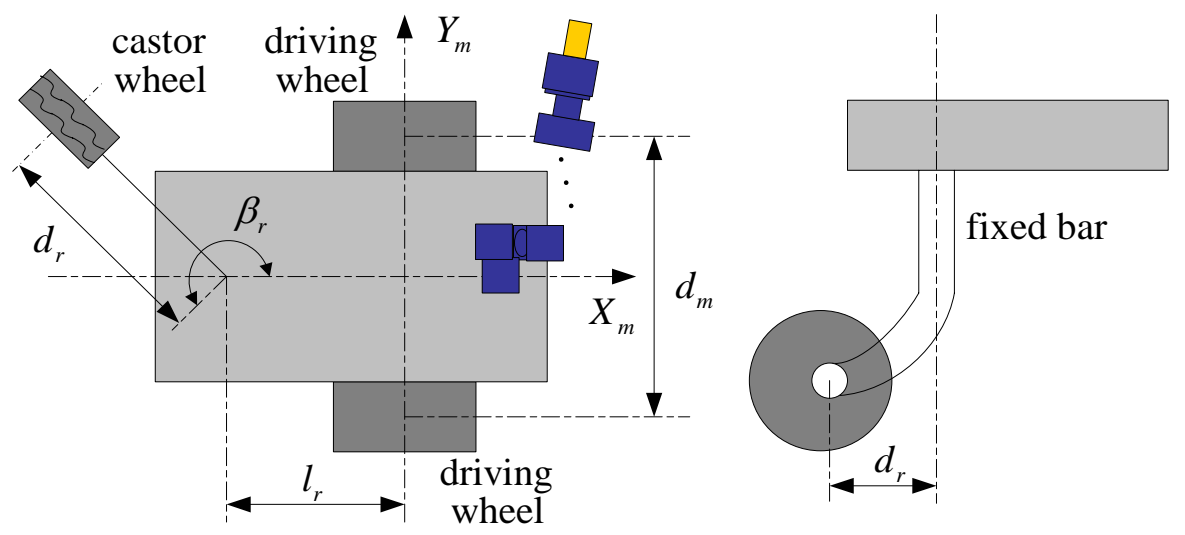

Fig. 3. Structure for the castor wheel.

Kinematic energy for the onboard modular manipulator can be calculated by

$$
T_{M}=\sum_{i=0}^{n}\left(\frac{1}{2} \cdot v_{c i}^{T} \cdot m_{i} \cdot v_{c i}+\frac{1}{2} \cdot \omega_{i}^{T} \cdot I_{i} \cdot \omega_{i}\right)
$$

where $T_{M}$ denotes kinetic energy for the modular manipulator; $m_{i}$ and $I_{i}$ are mass and inertial moment matrix for the $i^{\text {th }}$ module respectively; $v_{c i}$ and $\omega_{i}$ represent the linear and angular velocities for the mass centre of the $i^{\text {th }}$ module. 
Potential energy for the onboard modular manipulator can be calculated by

$$
U_{M}=\sum_{i=0}^{n}\left\{m_{i} \cdot g^{T} \cdot p_{c i}\right\}
$$

where $g=\left[\begin{array}{lll}0 & 0 & 9.81\end{array}\right]^{T}$ and $p_{c i}=\left[\begin{array}{lll}x_{i}^{B} & y_{i}^{B} & z_{i}^{B}\end{array}\right]^{T}$.

Then, the Lagrange function can be given by

$$
L=T_{m}-U_{m}+T_{M}-U_{M}
$$

The constraint dynamics for the nonholonomic mobile modular manipulator can be determined by

$$
\left\{\begin{array}{l}
\frac{d}{d t}\left(\frac{\partial L}{\partial \dot{x}_{m}}\right)-\frac{\partial L}{\partial x_{m}}=\lambda_{1} \cos \phi_{m}+\lambda_{2} \cos \phi_{m}+\lambda_{3} \sin \phi_{m}+\lambda_{4} \cos \left(\beta_{r}+\phi_{m}\right)+\lambda_{5} \sin \left(\beta_{r}+\phi_{m}\right) \\
\frac{d}{d t}\left(\frac{\partial L}{\partial \dot{y}_{m}}\right)-\frac{\partial L}{\partial y_{m}}=\lambda_{1} \sin \phi_{m}+\lambda_{2} \sin \phi_{m}-\lambda_{3} \cos \phi_{m}+\lambda_{4} \sin \left(\beta_{r}+\phi_{m}\right)-\lambda_{5} \cos \left(\beta_{r}+\phi_{m}\right) \\
\frac{d}{d t}\left(\frac{\partial L}{\partial \dot{\phi}_{m}}\right)-\frac{\partial L}{\partial \phi_{m}}=-\lambda_{1} \frac{d_{m}}{2}+\lambda_{2} \frac{d_{m}}{2}-\lambda_{4} l_{r} \sin \left(\beta_{r}\right)+\lambda_{5}\left(l_{r} \cos \beta_{r}-d_{r}\right) \\
\frac{d}{d t}\left(\frac{\partial L}{\partial \dot{\phi}_{r}}\right)-\frac{\partial L}{\partial \phi_{r}}=-\lambda_{4} r_{r} \\
\frac{d}{d t}\left(\frac{\partial L}{\partial \dot{\beta}_{r}}\right)-\frac{\partial L}{\partial \beta_{r}}=-\lambda_{5} d_{r} \\
\frac{d}{d t}\left(\frac{\partial L}{\partial \dot{\phi}_{L}}\right)-\frac{\partial L}{\partial \phi_{L}}=-\lambda_{1} r_{f}+\tau_{L}+\frac{\partial x^{T}}{\partial \phi_{L}} F_{e x t} \\
\frac{d}{d t}\left(\frac{\partial L}{\partial \dot{\phi}_{R}}\right)-\frac{\partial L}{\partial \phi_{R}}=-\lambda_{2} r_{f}+\tau_{R}+\frac{\partial x^{T}}{\partial \phi_{R}} F_{e x t} \\
\frac{d}{d t}\left(\frac{\partial L}{\partial \dot{q}_{i}}\right)-\frac{\partial L}{\partial q_{i}}=\tau_{i}+\frac{\partial x^{T}}{\partial q_{i}} F_{e x t},(i=1, \cdots, n)
\end{array}\right.
$$

where $\lambda_{i}(i=1, \cdots, 5)$ are Lagrange multipliers associated with the nonholonomic constraints, and $F_{\text {ext }} \in \mathfrak{R}^{6}$ is a vector for external forces; $\tau_{L}, \tau_{R}, \tau_{i}$ represent driving torques of the left wheel, the right wheel and the $i^{\text {th }}$ joint for the onboard modular manipulator respectively.

Equation 29 can be written into the matrix form

$$
\frac{d}{d t}\left(\frac{\partial L}{\partial \dot{\zeta}}\right)^{T}-\left(\frac{\partial L}{\partial \varsigma}\right)^{T}=B(\varsigma) \cdot\left(\tau+J^{T} \cdot F_{e x t}\right)+C(\varsigma) \cdot \lambda
$$


where $B(\varsigma)=\left[\begin{array}{ll}0_{(n+2) \times 5} & I_{(n+2) \times(n+2)}\end{array}\right]^{T}, C(\varsigma)=\left[\begin{array}{ll}A(\xi) & 0_{5 \times n}\end{array}\right]^{T}, \quad \tau=\left[\begin{array}{lllll}\tau_{L} & \tau_{R} & \tau_{1} & \cdots & \tau_{n}\end{array}\right]^{T}$, $\lambda=\left[\begin{array}{lll}\lambda_{1} & \cdots & \lambda_{5}\end{array}\right]^{T}$.

Equation 30 can be rewritten into the following standard form

$$
M(\varsigma) \cdot \ddot{\varsigma}+V(\varsigma, \dot{\zeta}) \cdot \dot{\zeta}+G(\varsigma)=B(\varsigma) \cdot\left(\tau+J^{T} \cdot F_{e x t}\right)+C(\varsigma) \cdot \lambda
$$

where $M$ is the inertial matrix; $V$ represents the centrifugal and coriolis matrix; and $G$ denotes the gravitional force vector.

Remark 1: Matrix $M$ is symmetric and positive semi-definite, i.e. $M^{T}=M \geq 0$.

Remark 2: Matrix $\dot{M}-2 V$ is skew-symmetric, i.e., for any $r \in \mathfrak{R}^{n+7}, r^{T} \cdot(\dot{M}-2 V) \cdot r=0$.

From Eq. 20,

$$
\dot{\zeta}=\left[\begin{array}{cc}
S(\xi) & 0_{7 \times n} \\
0_{n \times 2} & I_{n \times n}
\end{array}\right] \cdot \dot{q}
$$

Substituting Eq. 32 and its derivative into Eq. 31 yields

$$
\bar{M}(\varsigma) \cdot \ddot{q}+\bar{V}(\varsigma, \dot{\varsigma}) \cdot \dot{q}+\bar{G}(\varsigma)=\bar{\tau}
$$

where $\bar{M}=\bar{S}^{T} \cdot M \cdot \bar{S}, \quad \bar{V}=\bar{S}^{T} \cdot(V \cdot \bar{S}+M \cdot \dot{\bar{S}}) ， \quad \bar{G}=\bar{S}^{T} \cdot G, \quad \bar{\tau}=\tau+J^{T} \cdot F_{\text {ext }} \quad ; \quad$ the terms $\bar{S}^{T} \cdot B=I_{(n+2) \times(n+2)}$ and $\bar{S}^{T} \cdot C \cdot \lambda=0_{n+2}$ are eliminated.

If $n<4$, there are some dead zones that the end-effector can not reach; on the other hand, if $n>4$ the entire mobile modular manipulator will be a redundant one; this two cases are beyond the disscussion of this chapter. Assume the Jacobian matrix is full rank, soving the differential kinematics $\dot{x}=J \cdot \dot{q}$ and its derivative, yields

$$
\dot{q}=J^{-1} \cdot \dot{x}, \quad \ddot{q}=J^{-1} \cdot \ddot{x}-J^{-1} \cdot \dot{J} \cdot J^{-1} \cdot \dot{x}
$$

Substituting Eq. 34 into Eq. 33 and left multiplying $J^{-T}$ yields

$$
\tilde{M}(\varsigma) \cdot \ddot{x}+\tilde{V}(\varsigma, \dot{\varsigma}) \cdot \dot{x}+\tilde{G}(\varsigma)=\tilde{\tau}
$$

where $\tilde{M}=J^{-T} \cdot \bar{M} \cdot J^{-1}, \tilde{V}=J^{-T} \cdot\left(\bar{V}-\bar{M} \cdot J^{-1} \cdot \dot{J}\right) \cdot J^{-1}, \tilde{G}=J^{-T} \cdot \bar{G}$ and $\tilde{\tau}=J^{-T} \cdot \bar{\tau}$.

Remark 3: Matrix $\tilde{M}$ is symmetric and positive definite, i.e., $\tilde{M}^{T}=\tilde{M}>0$.

Proof: From Eq. 33 and Eq. 35,

$$
\tilde{M}=J^{-T} \cdot \bar{S}^{T} \cdot M \cdot \bar{S} \cdot J^{-1}
$$

According to Remark 1,

$$
\begin{aligned}
& \tilde{M}^{T}=J^{-T} \cdot \bar{S}^{T} \cdot M^{T} \cdot \bar{S} \cdot J^{-1}=J^{-T} \cdot \bar{S}^{T} \cdot M \cdot \bar{S} \cdot J^{-1}=\tilde{M} \\
& \tilde{M}=J^{-T} \cdot \bar{S}^{T} \cdot M \cdot \bar{S} \cdot J^{-1}=\left(\bar{S} \cdot J^{-1}\right)^{T} \cdot M \cdot\left(\bar{S} \cdot J^{-1}\right)>0
\end{aligned}
$$


Remark 4: Matrix $\dot{\tilde{M}}-2 \tilde{V}$ is skew-symmetric, i.e., for any $r \in \mathfrak{R}^{n+7}, r^{T} \cdot(\dot{\tilde{M}}-2 \tilde{V}) \cdot r=0$.

Proof: From Eq. 33 and Eq. 35,

$$
J^{T} \cdot \tilde{M} \cdot J=\bar{S}^{T} \cdot M \cdot \bar{S}
$$

Differentiating Eq. 38 yields

$$
J^{T} \cdot \dot{\tilde{M}} \cdot J+2 J^{T} \cdot \tilde{M} \cdot \dot{J}=\bar{S}^{T} \cdot \dot{M} \cdot \bar{S}+2 \bar{S}^{T} \cdot M \cdot \dot{\bar{S}}
$$

Then

$$
\dot{\tilde{M}}=J^{-T} \cdot \bar{S}^{T} \cdot \dot{M} \cdot \bar{S} \cdot J^{-1}+2 J^{-T} \cdot \bar{S}^{T} \cdot M \cdot \dot{\bar{S}} \cdot J^{-1}-2 \tilde{M} \cdot \dot{J} \cdot J^{-1}
$$

From Eq. 33 and Eq. 35

$$
\tilde{V}=J^{-T} \cdot \bar{S}^{T} \cdot V \cdot \bar{S} \cdot J^{-1}+J^{-T} \cdot \bar{S}^{T} \cdot M \cdot \dot{\bar{S}} \cdot J^{-1}-\tilde{M} \cdot \dot{J} \cdot J^{-1}
$$

Subtracting Eq. 41 multiplied by 2 from Eq. 40 yields

$$
\dot{\tilde{M}}-2 \tilde{V}=J^{-T} \cdot \bar{S}^{T} \cdot(\dot{M}-2 V) \cdot \bar{S} \cdot J^{-1}
$$

According to Eq. 41

$$
r^{T} \cdot(\dot{\tilde{M}}-2 \tilde{V}) \cdot r=\left(\bar{S} \cdot J^{-1} \cdot r\right)^{T} \cdot(\dot{M}-2 V) \cdot\left(\bar{S} \cdot J^{-1} \cdot r\right)=0
$$

Remark 5: Matrices $\tilde{M}, \tilde{V}$ and $\tilde{G}$ are all bounded as long as $J$ keeps nonsingular.

Remark 6: The dynamic equation in Eq. 35 is linearity in parameters, i.e., for any $\chi \in \mathfrak{R}^{6}$ independent of the strucuture parameters $\Phi, \tilde{M} \ddot{\chi}+\tilde{V} \dot{\chi}+\tilde{G}=Y(\varsigma, \dot{q}, \ddot{\chi}, \dot{\chi}) \Phi$. Here $Y(\varsigma, \dot{q}, \ddot{\chi}, \dot{\chi})$ is also independent of $\Phi$.

\section{Robust Adaptive Controller Design}

\subsection{A model-based controller}

Assume $x_{d}, \dot{x}_{d}$ and $\ddot{x}_{d}$ are the desired end-effector positions, velocities and accelerations. If precise dynamic parameters can be obtained in advance, the model-based controller can be exploited, as shown in Fig. 4 and Eq. 44.

$$
\tau=J^{T} \cdot\left\{\tilde{M} \cdot\left[\ddot{x}_{d}+K_{D} \cdot\left(\dot{x}_{d}-\dot{x}\right)+K_{P} \cdot\left(x_{d}-x\right)\right]+\tilde{V} \cdot \dot{x}+\tilde{G}-F_{e x t}\right\}
$$

where $K_{P}>0$ and $K_{D}^{T}=K_{D}>0$ are proportional and differential gain matrices. 


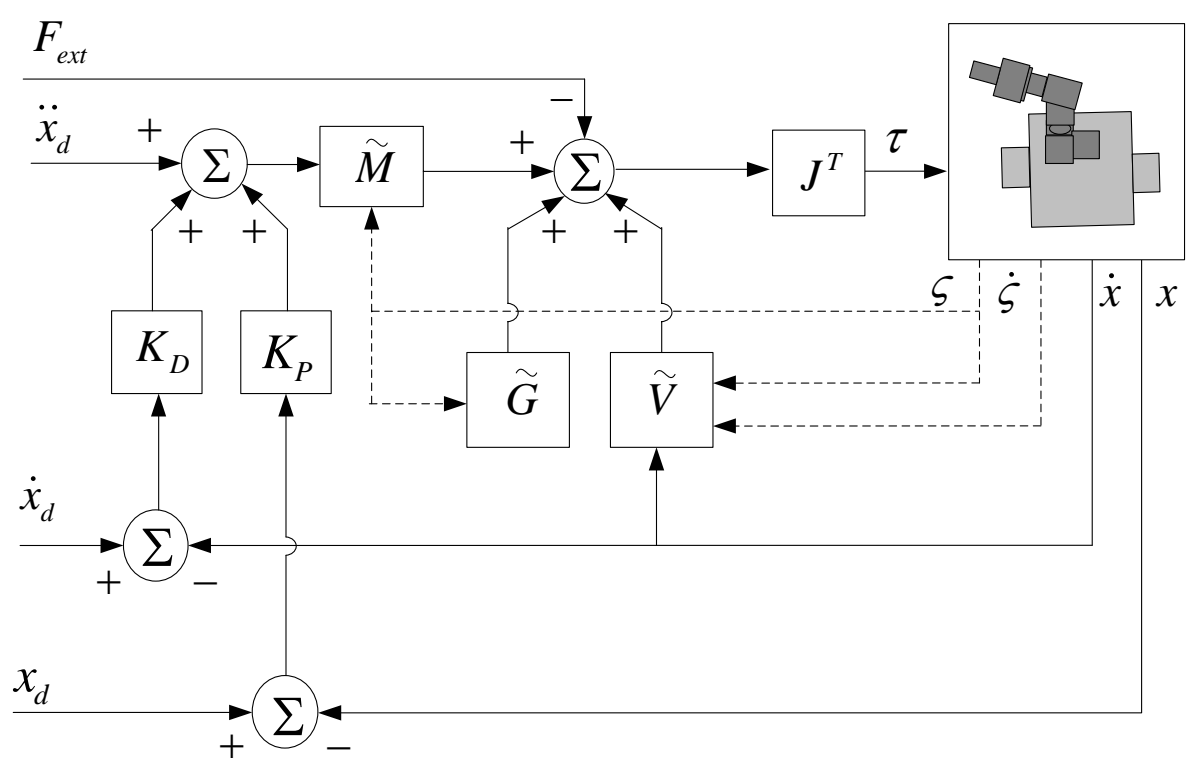

Fig. 4. A model-based controller.

Substituting Eq. 44 into Eq. 35 yileds

$$
\ddot{e}+K_{D} \cdot \dot{e}+K_{P} \cdot e=0
$$

where $e=x-x_{d}$ is the error vector.

Theorem 1: If $K_{P}>0$ and $K_{D}^{T}=K_{D}>0$, the system described in Eq. 45 is asymptotically stable, i.e., $e \rightarrow 0$ as $t \rightarrow \infty$.

Proof: Considering the nonnegative Lyapunov candidate as follows

$$
V_{S}=\frac{1}{2} \cdot \dot{e}^{T} \cdot \dot{e}+\frac{1}{2} \cdot e^{T} \cdot K_{P} \cdot e \geq 0
$$

The time derivative of Lyapunov candidate is

$$
\dot{V}_{S}=\dot{e}^{T} \cdot \ddot{e}+\dot{e}^{T} \cdot K_{P} \cdot e
$$

Substituting Eq. 45 into Eq. 47 yields

$$
\dot{V}_{S}=-\dot{e}^{T} \cdot K_{D} \cdot \dot{e} \leq 0
$$

From Eq. 46 and Eq. 48, $V_{S}$ is a Lyapunov function. Notice that when and only when $\dot{e}=0$, $\dot{V}_{S}=0$ may hold, so $\dot{e} \rightarrow 0$ as $t \rightarrow+\infty$. To analyze the steady-state error of the system, substituting $\dot{e}=0$ and $\ddot{e}=0$ into Eq. 45 yields $K_{P} \cdot e=0$, which is followed by $e=0$. That is to say, $e \rightarrow 0$ as $t \rightarrow+\infty$. That is all for the proof. 


\subsection{A robust adaptive controller}

To avoid measuring accelerations, the error system can be defined as follows

$$
\begin{aligned}
e(t) & =x(t)-x_{d}(t) \\
\dot{x}_{s}(t) & =\dot{x}_{d}(t)-\Lambda \cdot e(t) \\
s(t) & =\dot{x}(t)-\dot{x}_{s}(t)
\end{aligned}
$$

where $s(t)$ is the tracking error measure, and $\Lambda \in \mathfrak{R}^{6 \times 6}$ is a positive definite matrix.

From Eq. 49,

$$
\begin{aligned}
\dot{x}(t) & =s(t)+\dot{x}_{s}(t) \\
\ddot{x}_{s}(t) & =\ddot{x}_{d}(t)-\Lambda \cdot \dot{e}(t) \\
\ddot{x}(t) & =\dot{s}(t)+\ddot{x}_{s}(t)
\end{aligned}
$$

Substituting Eq. 50 into Eq. 35 yields

$$
\tilde{M} \cdot \dot{s}(t)+\tilde{V} \cdot s(t)+\tilde{M} \cdot \ddot{x}_{s}(t)+\tilde{V} \cdot \dot{x}_{s}(t)+\tilde{G}=\tilde{\tau}
$$

From Remark 6,

$$
\tilde{M} \cdot \ddot{x}_{s}(t)+\tilde{V} \cdot \dot{x}_{s}(t)+\tilde{G}=Y\left(\varsigma, \dot{q}, \ddot{x}_{s}, \dot{x}_{s}\right) \cdot \Phi
$$

Substituting Eq. 52 into Eq. 51 yields

$$
\tilde{M} \cdot \dot{s}(t)+\tilde{V} \cdot s(t)+Y\left(\varsigma, \dot{q}, \ddot{x}_{s}, \dot{x}_{s}\right) \cdot \Phi=\tilde{\tau}
$$

Let $\hat{\Phi}$ be the estimate of the structure parameter $\Phi$, the robust adaptive controller presented in this chapter is given by Eq. 54, and a control system diagram is shown in Fig. 5.

$$
\tau=J^{T} \cdot\left\{Y\left(\varsigma, \dot{q}, \ddot{x}_{s}, \dot{x}_{s}\right) \cdot \hat{\Phi}-K_{P} \cdot s(t)-K_{I} \cdot \int_{0}^{t} s(t) d t-\tau_{r}(s)-F_{\text {ext }}\right\}
$$

where the $1^{\text {st }}$ term forms an adaptive controller; the $2^{\text {nd }}$ and $3^{\text {rd }}$ terms form PI controller; $\tau_{r}(s)$ is the robust sliding mode term, with its element determined by

$$
\tau_{r i}\left(s_{i}\right)=k_{r i} \cdot e^{-\frac{1}{\left|s_{i}\right|}} \cdot \operatorname{sgn}\left(s_{i}\right)
$$

where $k_{r i}>0$ is a constant. 


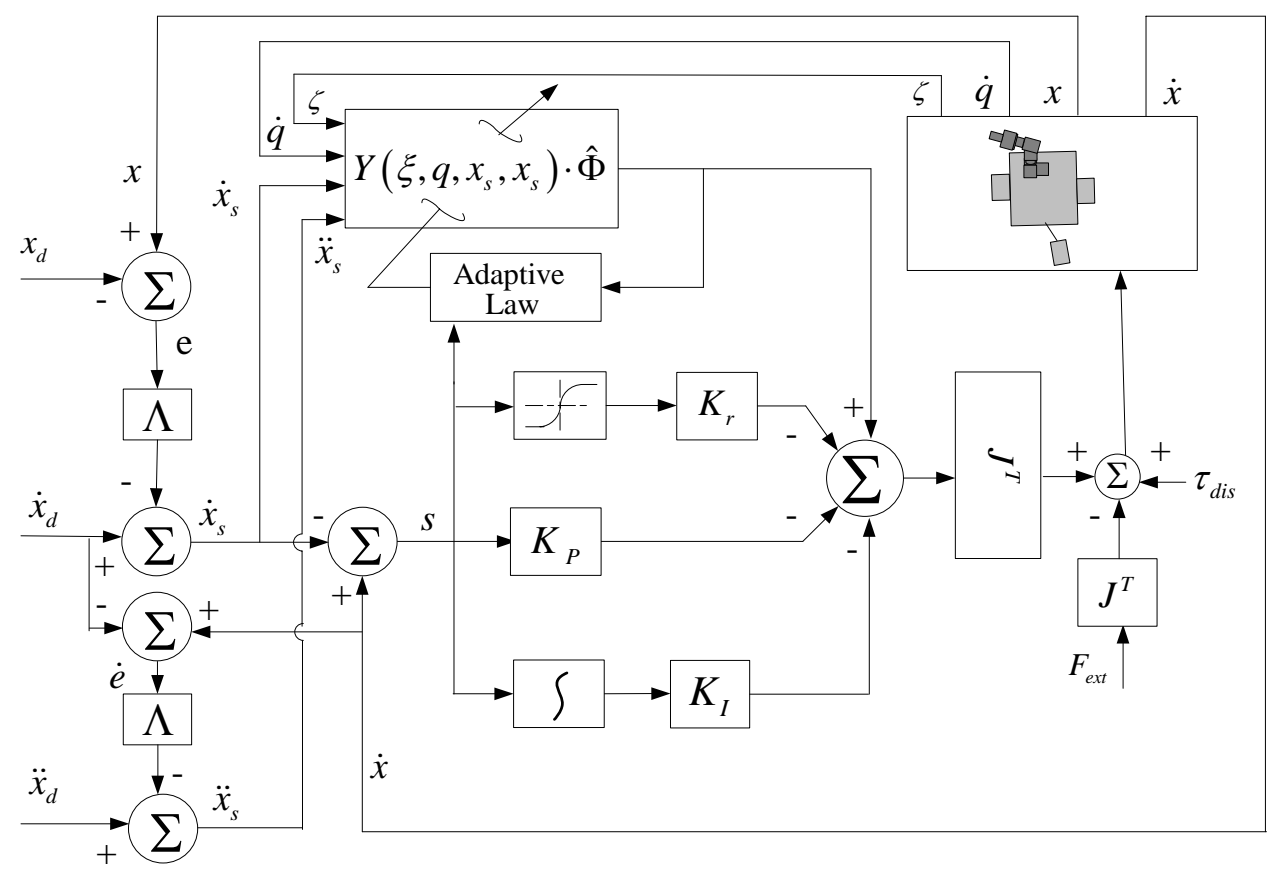

Fig. 5. A robint adantiven nontunl1nn

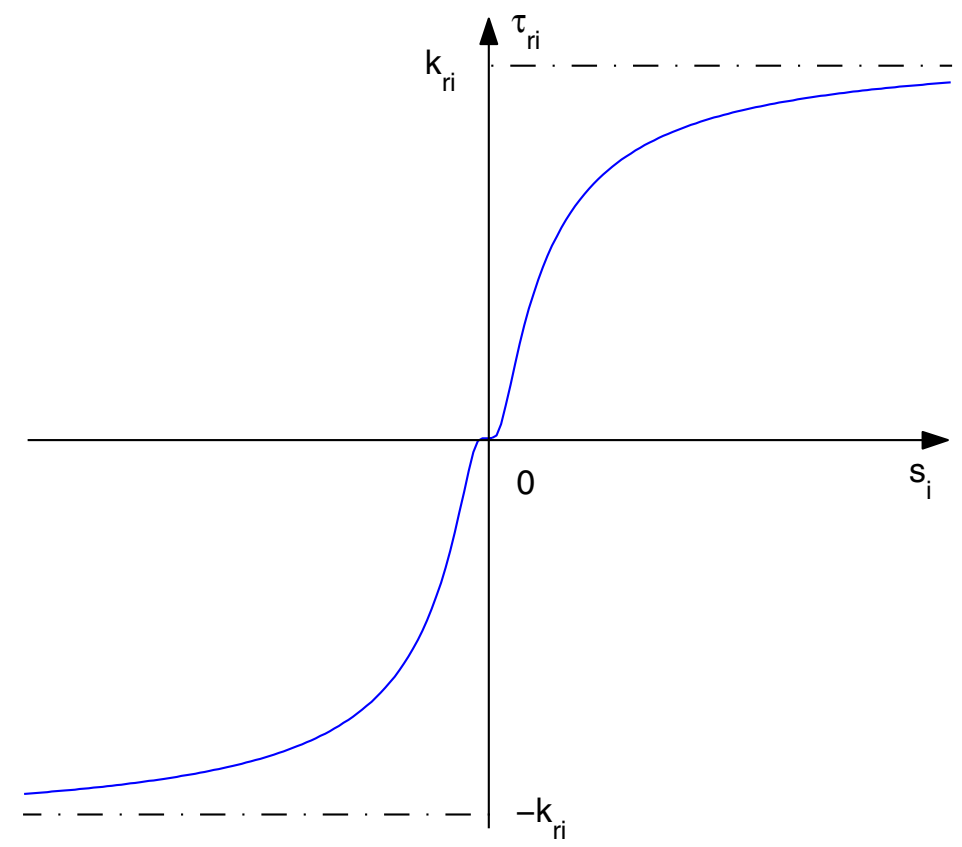

Fig. 6. A new sliding mode function. 
The sliding mode control law is not only continuous but also infinitely differentiable, which can eliminate the chattering caused by the classical sign function effectively. The output varies according to $\left|s_{i}\right|$. The funciton is plotted in Fig. 6 .

Substituting Eq. 54 into Eq. 53 and considering Eq. 35 at the same time yields

$$
\tilde{M} \cdot \dot{s}(t)+\tilde{V} \cdot s(t)+K_{P} \cdot s(t)+K_{I} \cdot \int_{0}^{t} s(t) d t+Y\left(\varsigma, \dot{q}, \ddot{x}_{s}, \dot{x}_{s}\right) \cdot(\Phi-\hat{\Phi})+\tau_{r}=0
$$

Theorem 2: The closed-loop system in Eq. 56 is asymptotically stable under the adaptation law by Eq. 57. The error signals are convergent with time, i.e., $e(t), \dot{e}(t) \rightarrow 0$ as $t \rightarrow \infty$. Furthermore, the signals in the system are all bounded.

$$
\dot{\hat{\Phi}}=-\Gamma \cdot Y^{T}\left(\varsigma, \dot{q}, \ddot{x}_{s}, \dot{x}_{s}\right) \cdot s
$$

where $\Gamma^{T}=\Gamma>0$ is a constant matrix.

Proof: Considering the nonnegative Lyapunov candidate as follows

$$
V_{S}=\frac{1}{2} \cdot\left[\int_{0}^{t} s(t) d t\right]^{T} \cdot K_{I} \cdot\left[\int_{0}^{t} s(t) d t\right]+\frac{1}{2} \cdot s^{T}(t) \cdot \tilde{M} \cdot s(t)+\frac{1}{2} \cdot \tilde{\Phi}^{T} \cdot \Gamma^{-1} \cdot \tilde{\Phi}
$$

where $\tilde{\Phi}=\hat{\Phi}-\Phi$.

Time derivative of the Lyapunov candidate is

$$
\dot{V}_{S}=s^{T} \cdot\left[\tilde{M} \cdot \dot{s}+K_{I} \cdot \int_{0}^{t} s(t) d t\right]+\frac{1}{2} \cdot s^{T} \cdot \dot{\tilde{M}} \cdot s+\dot{\tilde{\Phi}}^{T} \cdot \Gamma^{-1} \cdot \tilde{\Phi}
$$

From Eq. 56, we can obtain

$$
s^{T}\left[\tilde{M} \cdot \dot{s}+K_{I} \int_{0}^{t} s(t) d t\right]=-s^{T} \cdot \tilde{V} \cdot s-s^{T} \cdot K_{P} \cdot s+s^{T} \cdot Y\left(\varsigma, \dot{q}, \ddot{x}_{s}, \dot{x}_{s}\right) \tilde{\Phi}-s^{T} \tau_{r}
$$

Substituting Eq. 60 into Eq. 59 and considering Remark 4 at the same time yields

$$
\dot{V}_{S}=-s^{T} \cdot K_{P} \cdot s+s^{T} \cdot Y\left(\varsigma, \dot{q}, \ddot{x}_{s}, \dot{x}_{s}\right) \cdot \tilde{\Phi}-s^{T} \cdot \tau_{r}+\dot{\tilde{\Phi}}^{T} \cdot \Gamma^{-1} \cdot \tilde{\Phi}
$$

Considering that $\dot{\tilde{\Phi}}=\dot{\hat{\Phi}}$, substituting Eq. 57 into Eq. 61 yields

$$
\dot{V}_{S}=-s^{T} \cdot K_{P} \cdot s-s^{T} \cdot \tau_{r}
$$

Substituting Eq. 55 into Eq. 62 yields

$$
\dot{V}_{S}=-s^{T} \cdot K_{P} \cdot s-\sum_{i=1}^{6}\left\{k_{r i} \cdot e^{-\frac{1}{\left|s_{i}\right|}} \cdot s_{i} \cdot \operatorname{sgn}\left(s_{i}\right)\right\} \leq 0
$$

According to LaSalle's theorem, we can conclude that the system is asymptotically stable and $s \rightarrow 0$ as $t \rightarrow+\infty$. Let $\ell_{p}$ is the $p$-norm space, from Eq. 58 and Eq. 63, $s(t) \in \ell_{2}$. From 
Eq. 50, $e(t) \in \ell_{2} \cap \ell_{\infty}, \dot{e}(t) \in \ell_{2}$ and $e(t) \rightarrow 0$ as $t \rightarrow+\infty$. Because $V_{S} \geq 0$ and $\dot{V}_{S} \leq 0, \tilde{\Phi} \in \ell_{\infty}$ can be obtained; from Remark 5, $\Phi \in \ell_{\infty}$, so $\hat{\Phi} \in \ell_{\infty}$; therefore from the error equation, we can conclude that $\dot{s}(t) \in \ell_{\infty}$. Since $s(t) \in \ell_{2}$ and $\dot{s}(t) \in \ell_{\infty}, s \rightarrow 0$ as $t \rightarrow+\infty$, which is followed by $\dot{e}(t) \rightarrow 0$. This is the end of the proof.

\section{Simulation Results}

The mobile modular manipulator exploited in this simulation is made up of a 4-DOF onboard modular manipulator and 3-wheeled nonholonomic mobile platform, as shown in Fig. 1(a). The modular manipulator consists of 4 rotation modules named PowerCube produced by the AmtecGmbH Corporation of Germany. The Pioneer3-DXe produced by ActiveMedia Corporation of USA is used as the mobile platform.

According to the Denavit-Hartenberg notion, the simplified model of the mobile modular manipulator is drawn in Fig. 7.

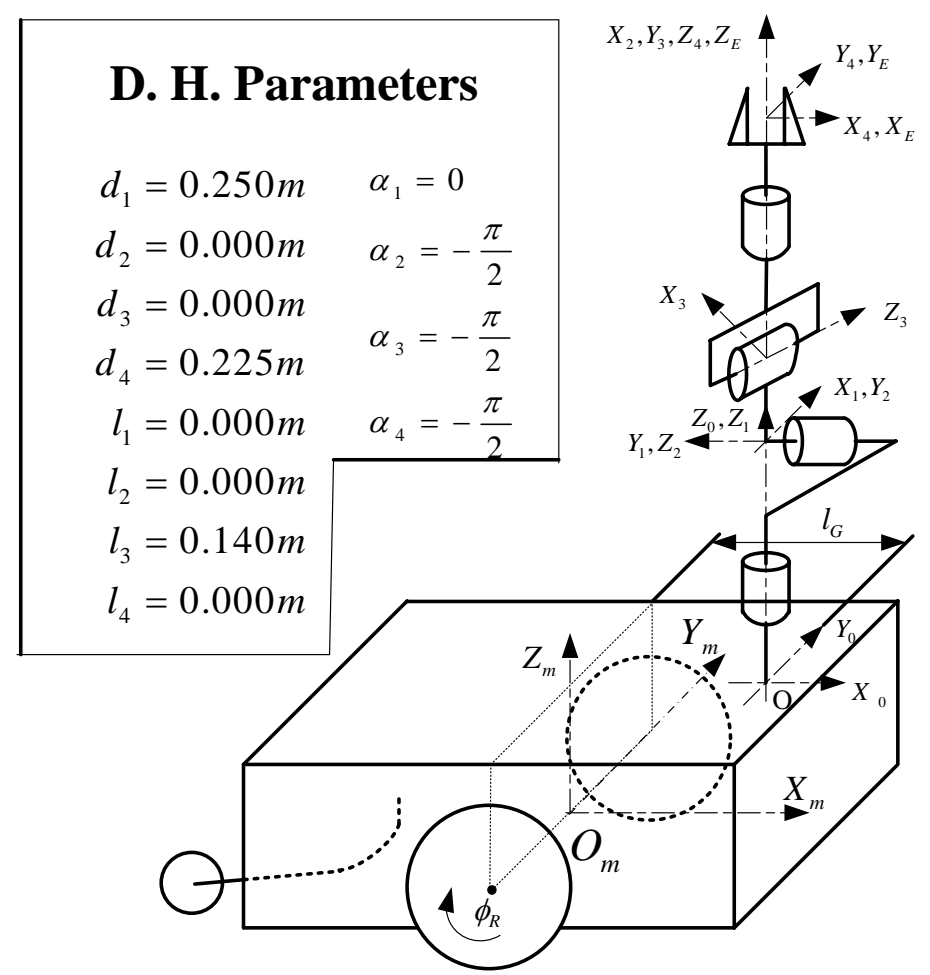

Fig. 7. A simplified model for the mobile modular manipulator.

Because of page limitations, the kinematics and dynamics model will not be detailed.

To ensure the controllers valid, the desired end-effector trajectory should be selected as far away from singularities. The Jacobian matrix is derived from Eq. 20 as follows 


$$
J=\left[\begin{array}{cccccc}
J_{11} & J_{12} & J_{13} & J_{14} & J_{15} & 0 \\
J_{21} & J_{22} & J_{23} & J_{24} & J_{25} & 0 \\
0 & 0 & 0 & -l_{3} \cos q_{2}+d_{4} \cos q_{2} \sin q_{3} & d_{4} \sin q_{2} \cos q_{3} & 0 \\
-\frac{r_{f}}{d_{m}} & \frac{r_{f}}{d_{m}} & 1 & \frac{\sin q_{2} \sin q_{3} \cos q_{3}}{\cos ^{2} q_{3}+\cos ^{2} q_{2} \sin ^{2} q_{3}} & \frac{-\cos q_{2}}{\cos ^{2} q_{3}+\cos ^{2} q_{2} \sin ^{2} q_{3}} & 0 \\
0 & 0 & 0 & \frac{-\cos q_{2} \sin q_{3}}{\sqrt{\cos ^{2} q_{3}+\cos ^{2} q_{2} \sin ^{2} q_{3}}} & \frac{-\sin q_{2} \cos q_{3}}{\sqrt{\cos ^{2} q_{3}+\cos ^{2} q_{2} \sin ^{2} q_{3}}} & 0 \\
0 & 0 & 0 & \frac{-\cos q_{3}}{\cos ^{2} q_{2}+\sin ^{2} q_{2} \cos ^{2} q_{3}} & \frac{\sin _{2} \cos q_{2} \sin q_{3}}{\cos ^{2} q_{2}+\sin ^{2} q_{2} \cos ^{2} q_{3}} & 1
\end{array}\right]
$$

where

$$
\begin{aligned}
& J_{11}=\frac{r_{f}}{2} \cdot \cos \phi_{m}-\frac{r_{f}}{d_{m}} \cdot\left(J_{13}-l_{G} \cdot \sin \phi_{m}\right), J_{21}=\frac{r_{f}}{2} \cdot \sin \phi_{m}-\frac{r_{f}}{d_{m}} \cdot\left(J_{23}+l_{G} \cdot \cos \phi_{m}\right), \\
& J_{12}=\frac{r_{f}}{2} \cdot \cos \phi_{m}+\frac{r_{f}}{d_{m}} \cdot\left(J_{13}-l_{G} \cdot \sin \phi_{m}\right), J_{22}=\frac{r_{f}}{2} \cdot \sin \phi_{m}+\frac{r_{f}}{d_{m}} \cdot\left(J_{23}+l_{G} \cdot \cos \phi_{m}\right), \\
& J_{13}=-l_{3} \sin \left(q_{1}+\phi_{m}\right) \cos q_{2}+d_{4} \sin \left(q_{1}+\phi_{m}\right) \cos q_{2} \sin q_{3}+d_{4} \cos \left(q_{1}+\phi_{m}\right) \cos q_{3}, \\
& J_{23}=+l_{3} \cos \left(q_{1}+\phi_{m}\right) \cos q_{2}-d_{4} \cos \left(q_{1}+\phi_{m}\right) \cos q_{2} \sin q_{3}+d_{4} \sin \left(q_{1}+\phi_{m}\right) \cos q_{3}, \\
& J_{14}=-l_{3} \cdot \cos \left(q_{1}+\phi_{m}\right) \cdot \sin q_{2}+d_{4} \cdot \cos \left(q_{1}+\phi_{m}\right) \cdot \sin q_{2} \cdot \sin q_{3}, \\
& J_{24}=-l_{3} \cdot \sin \left(q_{1}+\phi_{m}\right) \cdot \sin q_{2}+d_{4} \cdot \sin \left(q_{1}+\phi_{m}\right) \cdot \sin q_{2} \cdot \sin q_{3}, \\
& J_{15}=-d_{4} \cdot \cos \left(q_{1}+\phi_{m}\right) \cdot \cos q_{2} \cdot \cos q_{3}-d_{4} \cdot \sin \left(q_{1}+\phi_{m}\right) \cdot \sin q_{3}, \\
& J_{25}=-d_{4} \cdot \sin \left(q_{1}+\phi_{m}\right) \cdot \cos q_{2} \cdot \cos q_{3}+d_{4} \cdot \cos \left(q_{1}+\phi_{m}\right) \cdot \sin q_{3} .
\end{aligned}
$$

The determinant of Jacobian matrix is

$$
\operatorname{det}(J)=\frac{r_{f}^{2} \cdot l_{G} \cdot l_{3} \cdot \sin q_{2} \cdot \cos q_{2} \cdot \cos q_{3}}{d_{m} \cdot \sqrt{\cos ^{2} q_{3}+\cos ^{2} q_{2} \cdot \sin ^{2} q_{3}}}
$$

So, to avoid singularities, the boundary of joint angles in the joint space can be selected as follows

$$
\Omega=\left\{\phi_{L}, \phi_{R}, q_{1}, q_{2}, q_{3}, q_{4} \mid 0<q_{2}<\frac{\pi}{2}, \quad-\frac{\pi}{2}<q_{3}<\frac{\pi}{2}\right\}
$$

Simulations are conducted for two control schemes, i.e., the model-based controller (MBC) and the robust adaptive controller (RAC). To examine the disturbance suppression characteristics, a series of disturbance torques are introduced. Both the MBC and the RAC are required to control the end-effector to follow a sine like spatial trajectory as shown in Fig. 8 , which has been planned to ensure the system far away from singularities. All the joints and velocities are initialized to be zeros, except that $q_{2}=\pi / 3$. Simulation time interval is selected as 10 seconds. The gain matrices for the RAC are selected as follows 


$$
\begin{gathered}
K_{P}=\operatorname{diag}\{100\}, \quad K_{I}=\operatorname{diag}\{10.0\}, \quad K_{r}=\operatorname{diag}\{10.0\}, \\
\Lambda=\operatorname{diag}\{2.0\}, \quad \Gamma=\operatorname{diag}\{0.1\} .
\end{gathered}
$$

For the MBC, nominal dynamic parameters are adopted, which are assumed to be deviated from the real values by $10 \%$; the gain matrices are selected as $K_{P}=\operatorname{diag}\{100\}, K_{D}=\operatorname{diag}\{10.0\}$. Simulation results are presented by Fig. 8-14. The desired and controlled locus for the MBC and the RAC are shown in Fig. 8(a) and Fig. 8(b); Figure 9(a) and Fig. 9(b) present the tracking position errors; tracking Euler angular errors are shown in Fig. 10(a) and Fig. 10(b); Figure 11(a) and Fig. 11(b) give the tracking linear velocity errors; tracking Euler angular velocity errors are given by Fig. 12(a) and Fig. 12(b); time-varying control torques for the $\mathrm{MBC}$ and the RAC are shown in Fig. 13 and Fig. 14.

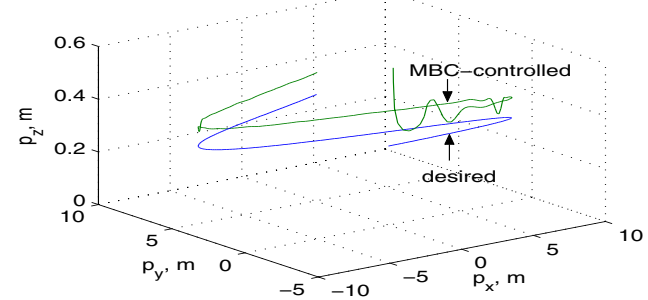

(a) Locus for the MBC

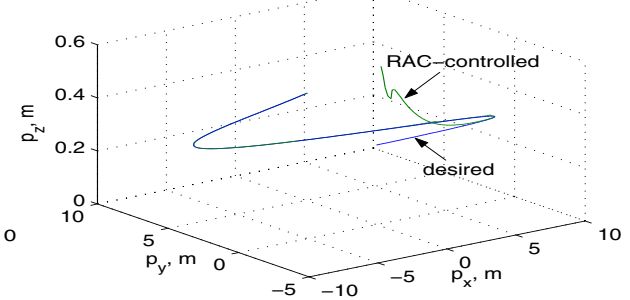

(b) Locus for the RAC

Fig. 8. Desired and controlled locus for the MBC and RAC.

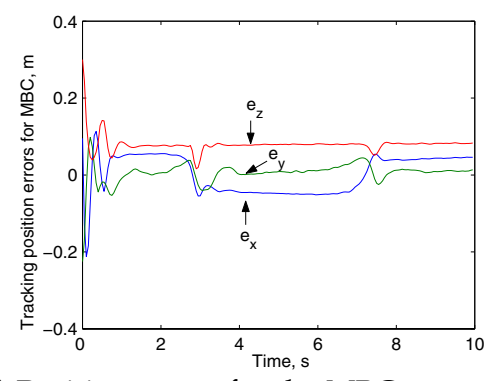

(a) Position errors for the MBC

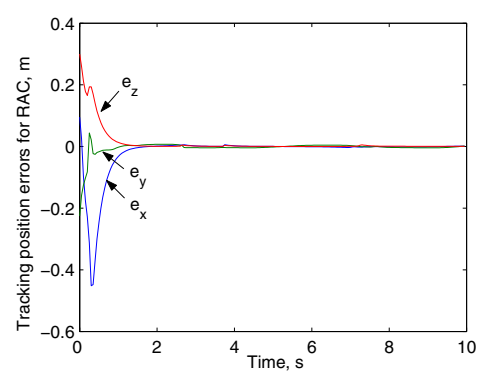

(b) Position errors for the RAC

Fig. 9. Tracking position errors for the MBC and RAC.

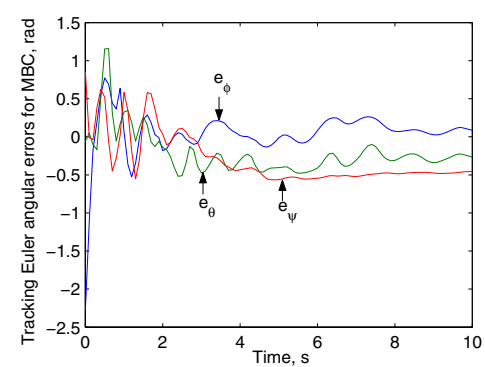

(a) Euler angular errors for MBC

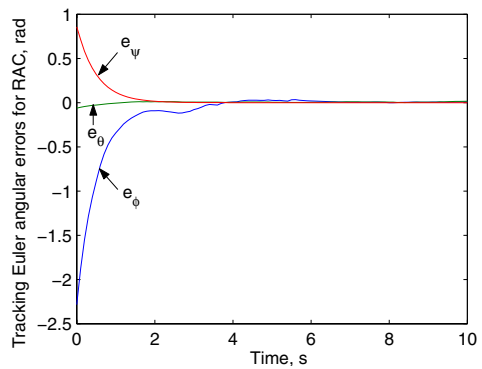

(b) Euler angular errors for RAC

Fig. 10. Tracking Euler angular errors for the MBC and RAC. 


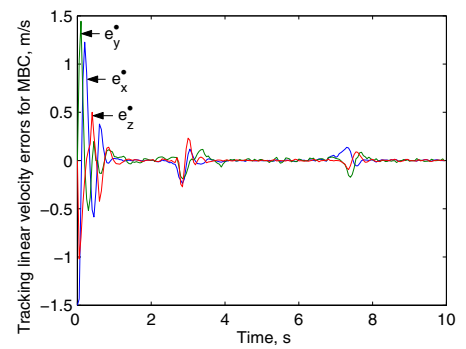

(a) Liner velocity errors for MBC

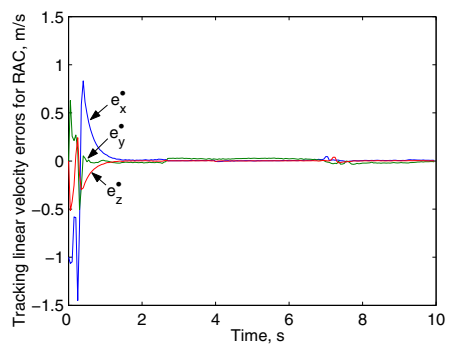

(b) Liner velocity errors for RAC

Fig. 11. Tracking linear velocity errors for the MBC and RAC.
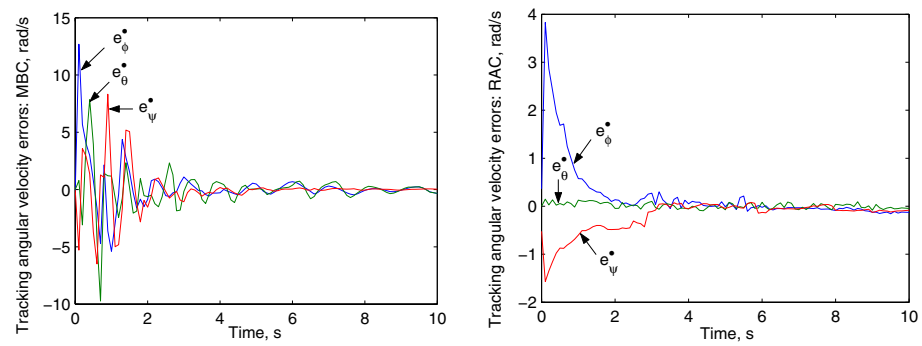

(a) Euler angular velocity errors for MBC (b) Euler angular velocity errors for RAC Fig. 12. Tracking Euler angular velocity errors for the MBC and RAC.
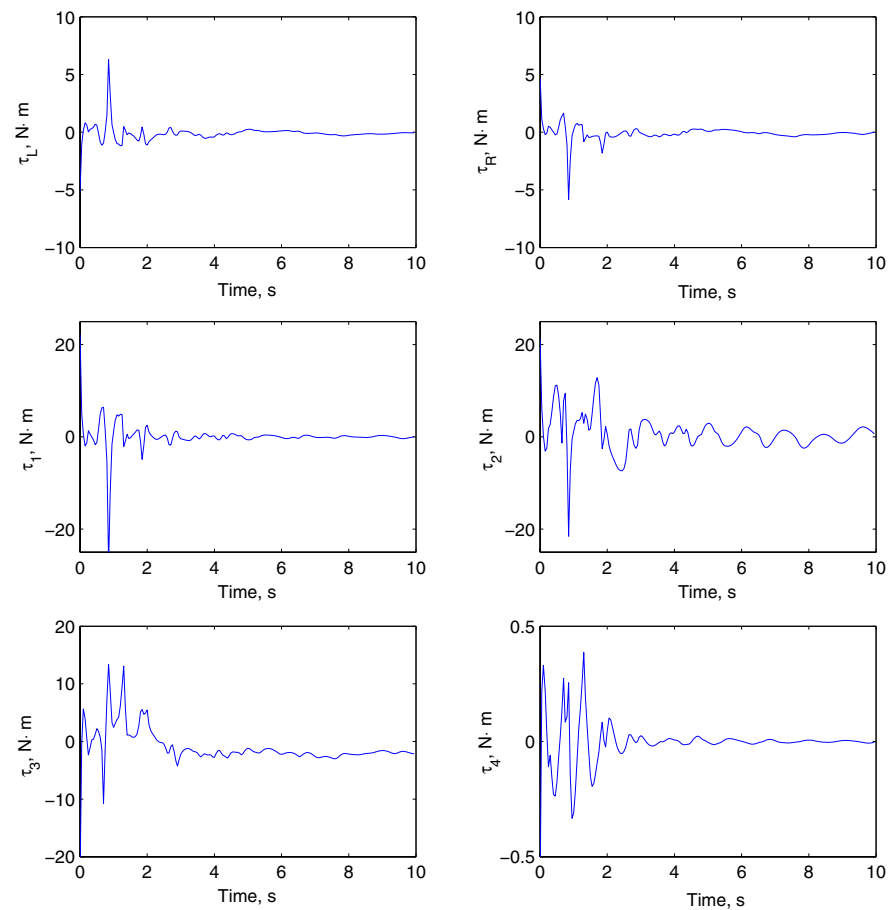

Fig. 13. Time-varying control torques for the MBC. 
Remark 7: From the simulation results, we can conclude that the proposed algorithms are effective to control the end-effector to follow some definite spatial trajectories. The robust adaptive controller behaves better than the model based controller, especially when parameter uncertainties and external disturbances exist.

\section{Conclusions}

In this chapter, 3-wheeled nonholonomic mobile modular manipulators are investigated. First, an integrated modelling method is proposed in consideration of the nonholonomic constraints and the interactive motions. Second, a model based controller and a robust adaptive controller are presented in task space directly. Third, simulations are carried out on a mobile modular manipulator composed of a 3-wheeled mobile platform and a 4-DOF onboard modular manipulator, and the simulation results demonstrate the effectiveness of the proposed algorithms. The proposed algorithms can be easily extended to some other mobile manipulators as well.
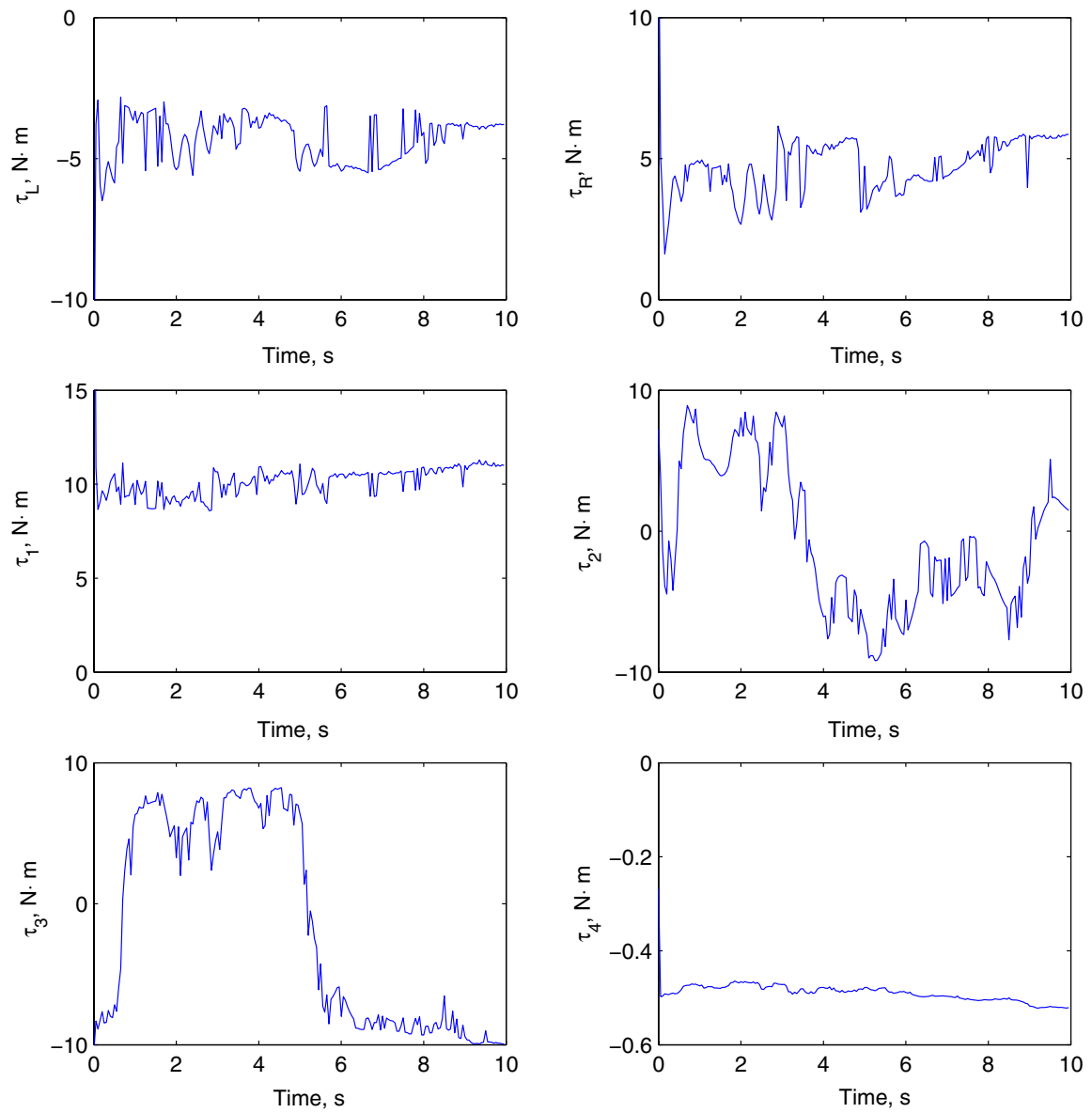

Fig. 14. Time-varying control torques for the RAC. 


\section{Future Research Work}

In this chapter, although the number of joints for the onboard modular manipulator is just assumed to be 4, the proposed method can be applied to m-DOF manipulator atop n-DOF mobile platform system in principle. The modelling and control for a redundant mobile modular manipulator is more challenging since self-motions have to be taken into consideration, (Li \& Liu, 2006b; Maciejewski \& Klein, 1989). The proposed algorithms are just verified by simulations, there is still a lot of work to do to verify the algorithms by real experiments. The task space information in this simulation is calculated via forward kinematics and differential kinematics, how to acquire task-space information directly and precisely is also a complex work, which needs deep research work on sensory technology and method.

\section{Acknowledgement}

The authors appreciate the fund support from the research committee of University of Macau under grant no.: RG082/04-05S/LYM/FST.

\section{References}

Campos, M.; Pereira, G.; Vale, S.; Bracarense, A.; Pinheiro, G. \& Oliveira, M. (2002). A mobile manipulator for installation and removal of aircraft warning spheres on aerial power transmission lines. Proceedings of IEEE International Conference on Robotics and Automation, pp. 3559-3564, Washington, DC, USA.

Carriker, W.; Khosla, P. \& Krogh, B. (1991). Path planning for mobile manipulators for multiple task execution. IEEE Trans. on Robotics and Automation, Vol. 7, pp. 403408.

Chen, I. \& Yang, G. (1998). Inverse kinematics for modular reconfigurable robots. Proceedings of IEEE International Conference on Robotics and Automation, pp. 1647-1652, Leuven, Belgium.

Chitta, S. \& Ostrowski, J. (2002). Motion planning for heterogeneous modular mobile systems. Proceedings of IEEE International Conference on Robotics and Automation, pp. 4077-4082, Washington, DC, USA.

Chung, J. \& Velinsky, S. (1999). Robust control of a mobile manipulator-dynamic modelling approach. Proceedings of the American Control Conference, pp. 2435-2439, San Diego, California, USA.

Colbaugh, R. (1998). Adaptive stabilization of mobile manipulators. Proceedings of the American Control Conference, pp. 1-5, Philadelphia, Pennsylvania, USA.

de Wit, C.; Siciliano, B. \& Bastin, G. (1996). Theory of Robot Control, Springer-Verlag.

Fei, Y.; Zhao, X. \& Song, L. (2001). A method for modular robots generating dynamics automatically. Robotica, Vol. 19, pp. 59-66.

Fukuda, T.; Nakagawa, S.; Kawauchi, Y. \& Buss, M. (1989). Structure decision method for self organizing robots based on cell structures-cebot. Proceedings of IEEE International Conference on Robotics and Automation, pp. 695-700, Scottsdale, AZ, USA.

Furuno, S.; Yamamoto, M. \& Mohri, A. (2003). Trajectory planning of mobile manipulator with stability considerations. Proceedings of IEEE International Conference on Robotics and Automation, pp. 3403-3408, Taipei, Taiwan, China. 
Ge, S.; Hang, C. \& Woon, L. (1997). Adaptive neural network control of robot manipulators in task space. IEEE Trans. on Industrial Electronics, Vol. 44, No. 6, pp. 746-752.

Ghasempoor, A. \& Sepehri, N. (1995). A measure of machine stability for moving base manipulators. Proceedings of IEEE International Conference on Robotics and Automation, pp. 2249-2254, Nagoya, Aichi, Japan.

Jamisola, R.; Ang, M.; Oetomo, D.; Khatib, O.; Lim, T. \& Lim, S. (2002). The operational space formulation implementation to aircraft canopy polishing using a mobile manipulator. Proceedings of IEEE International Conference on Robotics and Automation, pp. 400-405, Washington, DC, USA.

Li, Y.; Liu, Y.; Liu, X. \& Peng, Z. (2004a). Parameter identification and vibration control in modular manipulators. IEEE/ASME Trans. on Mechatronics, Vol. 9, No. 4, pp. 700705.

Li, Y. \& Liu, Y. (2004b). Control of a mobile modular manipulator moving on a slope. Proceedings of IEEE International Conference on Mechatronics, pp. 135-140, Istanbul, Turkey.

Liu, Y. \& Li, Y. (2005a). Dynamics and model-based control for mobile modular manipulators. Robotica, Vol. 23, No. 6, pp. 795-797.

Li, Y. \& Liu, Y. (2005b). Kinematics and tip-over stability analysis for the mobile modular manipulator. Proceedings of the Institution of Mechanical Engineers Part C: Journal of Mechanical Engineering Science, Vol. 219, No. 3, pp. 331-343.

Li, Y.; Liu, Y. \& Yan, S. (2005c). Adaptive Neural-Network Control for Redundant Nonholonomic Mobile Modular Manipulators, Advances in Neural Networks, Eds. by J. Wang, X. Liao and Z. Yi, Springer, LNCS 3498, Part III, pp.271-276.

Li, Y. \& Liu, Y. (2005d). Obstacle Avoidance for Redundant Nonholonomic Mobile Modular Manipulators via Neural Fuzzy Approaches. Advances in Natural Computation, Eds by L. Wang, K. Chen and Y.S. Ong, Springer, LNCS 3612, pp.1109-1118.

Li, Y. \& Liu, Y. (2006a). Online fuzzy logic control for tip-over avoidance of autonomous redundant mobile manipulators. International Journal of Vehicle Autonomous Systems, Vol.4, No.1, pp.24-43, 2006.

Li, Y. \& Liu, Y. (2006b). Real-time tip-over prevention and path following control for redundant nonholonomic mobile modular manipulators via fuzzy and neuralfuzzy approaches. ASME Trans. of Dynamic Systems, Measurement, and Control, (to appear).

Maciejewski, A. A. \& Klein, C. (1989). The Singular Value Decomposition: Computation and Application to Robotics. International Journal of Robotics Research, Vol. 8, No. 6, pp. 63-79.

Melek, W. \& Goldenberg, A. (2003). Neurofuzzy control of modular and reconfigurable robots. IEEE/ASME Trans. on Mechatronics, Vol. 8, No. 3, pp. 381-389.

Nagatani, K.; Hirayama, T.; Gofuku, A. \& Tanaka, Y. (2002). Motion planning for mobile manipulator with keeping manipulability. Proceedings of IEEE/RSJ International Conference on Intelligent Robotics and Systems, pp. 1663-1668, EPFL, Swizerland.

Papadopoulos, E. \& Rey, D. (1996). A new measure of tipover stability margin for mobile manipulators. Proceedings of IEEE International Conference on Robotics and Automation, pp. 3111-3116, Minneapolis, MN, USA.

Rus, D.; Donald, B. \& Jennings, J. (1995). Moving furniture with teams of autonomous robots. Proceedings of IEEE/RSJ International Conference on Intelligent Robotics and Systems, pp. 235-242, Pittsburgh, USA. 
Shen, W.; Salemi, B. \& Will, P. (2002). Hormone-inspired adaptive communication and distributed control for conro self-reconfigurable robots. IEEE Trans. on Robotics and Automation, Vol. 18, No. 5, pp. 700-712.

Stilwell, D. \& Bay, J. (1993). Toward the development of a material transport system using swarms of ant-like robots. Proceedings of IEEE International Conference on Robotics and Automation, pp. 766-771, Atlanta, Georgia, USA.

Stoy, K.; Shen, W. \& Will, P. (2002). Using role-based control to produce locomotion in chain-type self-reconfigurable robots. IEEE/ASME Trans. on Mechatronics, Vol. 7, No. 4, pp. 410-417.

Tanner, H. \& Kyriakopoulos, K. (2001). Mobile manipulator modelling with Kane's approach. Robotica, Vol. 19, pp. 675-690.

Tomita, T.; Murata, S.; Kurokawa, H.; Yoshida, E. \& Kokaji, S. (1999). Self-assembly selfrepair method for a distributed mechanical system. IEEE Trans. on Robotics and Automation, Vol. 15, No. 6, pp. 1035-1045.

Will, P. \& Grossman, D. (1975). An experimental system for computer controlled mechanical assembly. IEEE Trans. on Computer, Vol. 24, pp. 879-888.

Yamamoto, Y. \& Yun, X. (1996). Effect of the dynamic interaction on coordinated control of mobile manipulators. IEEE Trans. on Robotics and Automation, Vol. 12, No. 5, pp. 816-824.

$\mathrm{Yu}, \mathrm{Q}$. \& Chen, I. (2002). A general approach to the dynamics of nonholonomic mobile manipulator systems. ASME Trans. of Dynamic Systems, Measurement, and Control, Vol. 124, No. 4, pp. 512-521. 


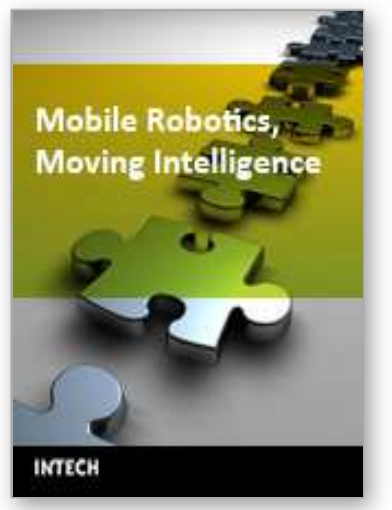

\author{
Mobile Robotics, Moving Intelligence \\ Edited by Jonas Buchli
}

ISBN 3-86611-284-X

Hard cover, 586 pages

Publisher Pro Literatur Verlag, Germany / ARS, Austria

Published online 01, December, 2006

Published in print edition December, 2006

This book covers many aspects of the exciting research in mobile robotics. It deals with different aspects of the control problem, especially also under uncertainty and faults. Mechanical design issues are discussed along with new sensor and actuator concepts. Games like soccer are a good example which comprise many of the aforementioned challenges in a single comprehensive and in the same time entertaining framework. Thus, the book comprises contributions dealing with aspects of the Robotcup competition. The reader will get a feel how the problems cover virtually all engineering disciplines ranging from theoretical research to very application specific work. In addition interesting problems for physics and mathematics arises out of such research. We hope this book will be an inspiring source of knowledge and ideas, stimulating further research in this exciting field. The promises and possible benefits of such efforts are manifold, they range from new transportation systems, intelligent cars to flexible assistants in factories and construction sites, over service robot which assist and support us in daily live, all the way to the possibility for efficient help for impaired and advances in prosthetics.

\title{
How to reference
}

In order to correctly reference this scholarly work, feel free to copy and paste the following:

Yangmin Li and Yugang Liu (2006). Dynamics and Control for Nonholonomic Mobile Modular Manipulators, Mobile Robotics, Moving Intelligence, Jonas Buchli (Ed.), ISBN: 3-86611-284-X, InTech, Available from: http://www.intechopen.com/books/mobile_robotics_moving_intelligence/dynamics_and_control_for_nonholono mic_mobile_modular_manipulators

\section{INTECH}

open science | open minds

\section{InTech Europe}

University Campus STeP Ri

Slavka Krautzeka 83/A

51000 Rijeka, Croatia

Phone: +385 (51) 770447

Fax: +385 (51) 686166

www.intechopen.com

\section{InTech China}

Unit 405, Office Block, Hotel Equatorial Shanghai

No.65, Yan An Road (West), Shanghai, 200040, China 中国上海市延安西路65号上海国际贵都大饭店办公楼405单元

Phone: +86-21-62489820

Fax: +86-21-62489821 
(C) 2006 The Author(s). Licensee IntechOpen. This chapter is distributed under the terms of the Creative Commons Attribution-NonCommercialShareAlike-3.0 License, which permits use, distribution and reproduction for non-commercial purposes, provided the original is properly cited and derivative works building on this content are distributed under the same license. 